Pacific Journal of Mathematic

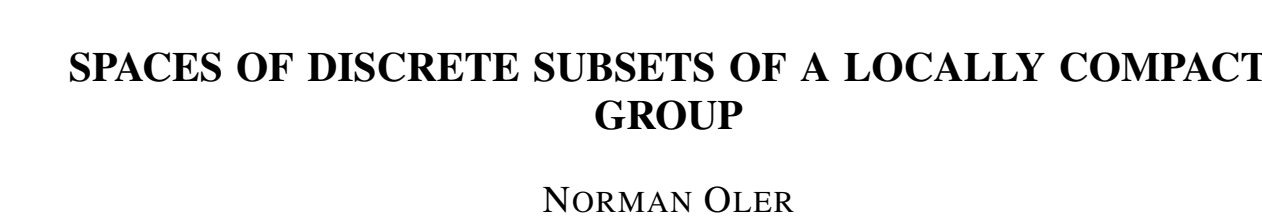




\title{
SPACES OF DISCRETE SUBSETS OF A LOCALLY COMPACT GROUP
}

\author{
N. OLER
}

This paper represents a continuing effort to develop elements of a general theory of packing and covering by translates of a fixed subset of a group. For $P$ a subset of a group $X$ a subset $L$ is a left $P$-packing if for any distinct elements $x_{1}$ and $x_{2}$ in $L, x_{1} P \cap x_{2} P$ is empty. A subset $M$ of $X$ is a left $P$-covering if $M P=X$. The Chabauty topology on the set of discrete subgroups of a locally compact group has been used only to a rather limited extent in the Geometry of Numbers but by its very definition is a natural one to work with in studying packing and covering problems. The main results of this paper are that the Chabauty topology extends to the family of all closed discrete subsets containing the identity and that if $X$ is $\sigma$-compact then $S(X, P)$ the space of all left $P$-packings for a fixed neighborhood $P$ of the identity is locally compact.

The Chabauty topology [1] extends in the following way. Let $X$ be a Hausdorff locally compact group with identity $e$ and $\mathscr{N}$ the family of open neighborhoods of $e$. We introduce the following notation: For any three subsets $A, B$ and $C$ of $X$ we define $L(A, B, C)$ to be the set of subsets, $A^{\prime}$, of $X$ satisfying

$$
A^{\prime} \cap B \subset A C \text { and } A \cap B \subset A^{\prime} C
$$

and $R(A, B, C)$ as the set of subsets $A^{\prime}$ of $X$ satisfying

$$
A^{\prime} \cap B \subset C A \text { and } A \cap B \subset C A^{\prime} \text {. }
$$

DEFINITION 1. We denote by $\mathscr{S}(X)$ the space of closed discrete subsets of $X$ containing $e$ with the topology generated by $\{L(H, K, U)$ : $H \in \mathscr{S}(X) ; K$ a compact subset of $X ; U \in \mathscr{N}\}$.

The following two assertions are immediate consequences of the definition of $L$.

Proposition 1. If $U_{1} \supset U_{2}$ then $L\left(H, K, U_{1}\right) \supset L\left(H, K, U_{2}\right)$.

Proposition 2. If $K_{1} \supset K_{2}$ then $L\left(H, K_{1} U\right) \subset\left(H, K_{2}, U\right)$.

As a further consequence we have 
Proposition 3. $\{L(H, K, U): K$ compact; $U \in \mathscr{N}\}$ is a local basis.

Proof. $L\left(H, K_{1} \cup K_{2}, U_{1} \cap U_{2}\right) \subset L\left(H, K_{1}, U_{1}\right) \cap L\left(H, K_{2}, U_{2}\right)$.

We shall require

Lemma. Given $H \in \mathscr{S}(X)$ and $K$ compact there exists $V \in \mathscr{N}$ such that $H V \cap K=(H \cap K) V$.

Proof. Choose $U$ a compact neighborhood of $e$ and consider the finite set $S=H \cap K U$. For $h \in S \backslash K$ there exists a symmetric $V_{h} \in \mathscr{N}$ with $V_{h} \subset U$ such that $h V_{h} \cap K=\varnothing$. Let $V=\bigcap V_{h}, h \in S \backslash K$ then $V$ has the asserted property. For $S V \cap K=\varnothing$ and if $h \in H \backslash S$ then $h \notin K U$ so $h \notin K V$ hence $h V \cap K=\varnothing$ since $V$ is symmetric. Thus for $h \in H$ we have that $h V \cap K \neq \varnothing$ if and only if $h \in K$ and the lemma follows.

Suppose now that $H_{1} \in L(H, K, W)$ then there exists $V \in \mathscr{T}, V$ relatively compact, $V \subset W$ for which $H_{1} \in L(H, K, V)$. Namely, if $h \in H \cap K$ then $h \in h_{1} W$ for some $h_{1} \in H$ so there exists $V_{h} \in \mathscr{N}, V_{h}$ relatively compact and $V_{h} \subset W$ such that $h \in h_{1} V_{h}$. Let $V^{\prime}=\bigcup V_{h}$, $h \in H \cap K$ then $H \cap K \subset H_{1} V^{\prime}$. Similarly there exists $V^{\prime \prime} \in \mathscr{N}$, $V^{\prime \prime}$ relatively compact and $V^{\prime \prime} \subset W$ such that $H_{1} \cap K \subset H V^{\prime \prime}$. With $V=V^{\prime} \cup V^{\prime \prime}$ the assertion follows.

If $k \in h_{1} V$ then $h_{1} \in k V^{-1}$ so that $H_{1} V \cap K \subset\left(H_{1} \cap K V^{-1}\right) V$ and therefore $H \cap K \subset\left(H_{1} \cap K V^{-1}\right) V$. Similarly $H_{1} \cap K \subset\left(H \cap K V^{-1}\right) V$. Let $h_{1} \in H_{1} \cap K$ then $h_{1} \in h^{\prime} V$ for some $h^{\prime} \in H \cap K V^{-1}$ so that $h^{\prime} \in$ $h_{1} V^{-1}$. There exists $V_{h_{1}} \in \mathscr{N}$ such that $h^{\prime} V_{h_{1}} \subset h_{1} V^{-1}$ and so for any $h^{\prime \prime} \in h^{\prime} V_{h_{1}}$ we have that $h_{1} \in h^{\prime \prime} V_{h_{1}}$. Let $V_{1}=\bigcap V_{h_{1}}, h_{1} \in H_{1} \cap K$.

The lemma provides that there exists $V_{2} \in \mathscr{N}$ such that $H_{1} V_{2} \cap$ $K=\left(H_{1} \cap K\right) V_{2}$.

Since $H_{1} \cap K \subset H V$ there exists $V_{3} \in \mathscr{N}$ such that $\left(H_{1} \cap K\right) V_{3} \subset$ $H V$.

Let $\quad U^{\prime}=V_{1} \cap V_{2} \cap V_{3}$ and $U=U^{\prime} \cap U^{\prime-1}$ and consider $H_{2} \in L\left(H_{1}\right.$, $\left.K \overline{V^{-1}}, U\right)$. Since $H_{1} \cap K \overline{V^{-1}} \subset H_{2} U$ there is for each $h^{\prime} \in H_{1} \cap K \overline{V^{-1}}$ an element $h_{2} \in H_{2}$ such that $h^{\prime} \in h_{2} U$, so $h_{2} \in h^{\prime} U$, hence $h_{2} \in h^{\prime} V_{1}$. It follows that $H \cap K \subset H_{2} V$ by the argument motivating the choice of $V_{1}$.

On the other hand $H_{2} \cap K \subset H_{2} \cap K \overline{V^{-1}} \subset H_{1} U$. Therefore $H_{2} \cap$ $K \subset H_{1} U \cap K \subset\left(H_{1} \cap K\right) U$ by choice of $V_{2}$. Our choice of $V_{3}$ now ensures that $\left(H_{1} \cap K\right) U \subset H V$. Hence $H_{2} \cap K \subset H V$. Thus $L\left(H_{1}, K \overline{V^{-1}}, U\right) \subset$ $L(H, K, V)$. Recalling Proposition 3, we have proved 
TheOREM 1. The family $\{L(H, K, V): K$ compact $V \subset \mathscr{N}\}$ is a basis for the topology on $\mathscr{S}(X)$.

As an immediate consequence in view of Proposition 1 and the lemma we have

Corollary. The family $\{L(H, K, V): K$ compact, $V$ relatively compact, symmetric and satisfying $H V \cap K=(H \cap K) V\}$ is a basis for the topology on $\mathscr{S}(X)$.

That $\mathscr{S}(X)$ is Hausdorff we see as follows.

Let $H$ and $H^{\prime}$ be distinct elements of $\mathscr{S}(X)$ then there exists $a \in H \Delta H^{\prime}$ say $a \in H \backslash H^{\prime}$. There exists a neighborhood $V \in \mathscr{N}$ which is symmetric and relatively compact for which $a V \cap H^{\prime}=\varnothing$. Choose a symmetric $V_{1} \in \mathscr{N}$ such that $V_{1}^{2} \subset V$ and consider the neighborhoods $L\left(H^{\prime}, a \bar{V}_{1}, V_{1}\right)$ and $L\left(H,\{a\}, V_{1}\right)$. Let $H_{1} \in L\left(H^{\prime}, a \bar{V}_{1}, V_{1}\right)$. Were $H_{1} \in L\left(H,\{a\}, V_{1}\right)$ then $H \cap\{a\} \subset H_{1} V_{1}$ i.e. $a \in H_{1} V_{1}$ say $a \in h_{1} V_{1}$ for some $h_{1} \in H_{1}$ implying that $h_{1} \in a V_{1}$. Now $H \cap a \bar{V}_{1} \subset H^{\prime} V_{1}$ hence $h_{1} \in H^{\prime} V_{1}$. Thus $a V_{1} \cap H^{\prime} V_{1} \neq \varnothing$ and therefore $H^{\prime} \cap a V_{1}^{2} \neq \varnothing$. But this implies that $a V \cap H^{\prime} \neq \varnothing$ which is a contradiction. It follows that $L\left(H^{\prime}, a \bar{V}_{1}, V_{1}\right) \cap L\left(H,\{a\}, V_{1}\right)=\varnothing$.

THEOREm 2. The topology on $\mathscr{S}(X)$ is equivalent to that generated by $\{R(H, K, V): H \in \mathscr{S}(X), K$ compact $V \in \mathscr{N}\}$.

Proof. Let $L(H, K, V)$ be such that $V$ is symmetric and $H V \cap$ $K=(H \cap K) V$.

By an analogous proof of the above lemma, there exists a symmetric $U \in \mathscr{N}$ satisfying $U H \cap K=U(H \cap K)$. Moreover $H \cap K$ being finite we can choose such a $U$ for which $U h \subset h V$ for all $h \in H \cap K$.

Let $\quad H_{1} \in R(H, K, U)$. Then $\quad H_{1} \cap K \subset U H \cap K \subset U(H \cap K) \subset$ $(H \cap K) V \subset H V$. Also $H \cap K \subset U H_{1} \cap K$ so that if $h \in H \cap K$ then $h \in U h_{1}$ for some $h_{1} \in H_{1}$; hence $h_{1} \in U h$ and so $h_{1} \in h V$. Hence $h \in h_{1} V$. It follows that $H \cap K \subset H_{1} V$. Thus $R(H, K, U) \subset L(H, K, V)$. By a similar argument for any $R(H, K, U)$ there exists $W \in \mathscr{N}$ such that $L(H, K, W) \subset R(H, K, U)$.

We turn now to the concept of packing.

Definition 2. For $V \in \mathscr{N}$, say that a set $H$ is a (left) $V$ packing if, for each pair of distinct elements $h_{1}$ and $h_{2}$ in $H, h_{1} V \cap$ $h_{2} V=\varnothing$. 
Definition 3. Say that a subset $H$ of $X$ is uniformly discrete if there exists $U \in \mathscr{N}$ such that $h U \cup H=\{h\}$ for each $h \in H$. Denote the class of those uniformly discrete subsets of $X$ which contain the identity by $\mathrm{S}(X)$.

The class of uniformly discrete subsets of $X$ clearly coincides with the class of left $V$-packing for all $V \in \mathscr{N}$. For if $h_{1} U \cap h_{2}=$ $\varnothing$ for any $h_{1} \neq h_{2}, h_{1}, h_{2} \in H$ then for any $V \in \mathscr{N}$ such that $V V^{-1} \subset$ $U$ we have $h_{1} V \cap h_{2} V=\varnothing$. Conversely $h_{1} V \cap h_{2} V=\varnothing$ implies that $h_{1} V V^{-1} \cap h_{2}=\varnothing$.

We now restrict ourselves to $S(X)$ with the relative topology induced by $\mathscr{S}(X)$. We remark, however, that in the sequel some of our results concerning $S(X)$ will be true also of $\mathscr{S}(X)$ in view of

Proposition 4. $S(X)$ is everywhere dense in $\mathscr{S}(X)$.

Proof. Suffice to observe that $H \cap K \in L(H, K, V)$.

Denoting by $p(K, V)$ the subset of $S(x) \times S(x)$ consisting of elements $\left(H_{1}, H_{2}\right)$ which satisfy $H_{1} \cap K \subset H_{2} V$ and $H_{2} \cap K \subset H_{1} V$ we see that the family $U=\{p(K, V): K$ compact, $V \in \mathscr{N}\}$ is the basis of a uniform structure. For we have that $p\left(K_{1} \cup K_{2}, V_{1} \cap V_{2}\right) \subset$ $p\left(K_{1}, V_{1}\right) \cap p\left(K_{2}, V_{2}\right)$, that each member of $U$ contains the diagonal and that $p(K, V) \in U$ implies $p(K, V)^{-1} \in U$. Further, if $W^{2} \subset V, W$ symmetric and $\bar{W}$ compact then $\left(H_{1}, H_{2}\right) \in p(K \bar{W}, W)$ and $\left(H_{2}, H_{3}\right) \in$ $p(K \bar{W}, W)$ implies that $H_{1} \cap K \subset H_{2} W \cap K$, so $H_{1} \cap K \subset\left(H_{2} \cap K W\right) W \subset$ $\left(H_{2} \cap K \bar{W}\right) W \subset H_{3} W$ and similarly $H_{3} \cap K \subset H_{1} W$. Thus $p(K \bar{W}, W) \circ$ $p(K \bar{W}, W) \subset p(K, V)$. Clearly $S(X)$ is the uniform topology induced by $U$ and therefore we have

Proposition 5. $S(X)$ is completely regular.

Suppose now that $K$ is compact, $V \in \mathscr{N}$ and $\bar{V}$ is compact. We shall show that $L(H, K V, \bar{V})$ is closed.

The complement $\mathscr{C} L(H, K V, \bar{V})$ consists of elements $H_{1}$ for which either of the following holds:

(i ) $H_{1} \cap K V \not \subset H \bar{V}$

(ii) $H \cap K V \not \subset H_{1} \bar{V}$.

In the case (i) there exists $h_{1} \in H_{1} \cap K V$ such that $h_{1} \notin H \bar{V}$ so $h_{1} \notin H \bar{V} \cap K \bar{V}$ hence $h_{1} \notin\left(H \cap K \bar{V} \bar{V}^{-1}\right) \bar{V}$. Since $H \cap K \bar{V} \bar{V}^{-1}$ is finite, $\left(H \cap K \bar{V} \bar{V}^{-1}\right) \bar{V}$ is compact and there exists a symmetric neighborhood $U \in \mathscr{N}$ such that $h_{1} U \cap\left(H \cap K \bar{V} \bar{V}^{-1}\right) \bar{V}=\varnothing$. Moreover we can choose $U$ so that $h_{1} U \subset K V$. If $H_{2} \in L\left(H_{1}, K \bar{V}, U\right)$ then $H_{1} \cap K \bar{V} \subset H_{2} U$ and there exists $h_{2} \in H_{2}$ for which $h_{1} \in h_{2} U$ hence 
$h_{2} \in h_{1} U$ and therefore $h_{2} \in K V$. On the other hand, were $H_{2} \in$ $L(H, K V, \bar{V})$ we would find $h_{2} \in H \bar{V} \cap K \bar{V}$ hence $h_{2} \in\left(H \cap K \bar{V} \bar{V}^{-1}\right) \bar{V}$ contradicting the fact that $h_{1} U \cap\left(H \cap K \bar{V} \bar{V}^{-1}\right) \bar{V}=\varnothing$. It follows that $L\left(H_{1}, K \bar{V}, U\right) \subset \mathscr{C} L(H, K V, \bar{V})$.

In the case (ii) there exists $h \in H \cap K V$ such that $h \notin H_{1} \bar{V} \cap K \bar{V}$ so $h \notin\left(H_{1} \cap K \bar{V} \bar{V}^{-1}\right) \bar{V}$. The latter set being compact, there exists a symmetric $U \in \mathscr{N}$ such that $U h \cap\left(H_{1} \cap K \bar{V} \bar{V}^{-1}\right) \bar{V}=\varnothing$. Furthermore we can choose $U$ small enough that $U H_{1} \cap K \bar{V} \bar{V}^{-1}=U\left(H_{1} \cap K \bar{V} \bar{V}^{-1}\right)$. Let $H_{2} \in R\left(H_{1}, K \bar{V} \bar{V}^{-1}, U\right)$ then

$$
H_{2} \cap K \bar{V} \bar{V}^{-1} \subset U H_{1} \cap K \bar{V} \bar{V}^{-1} .
$$

Were $H_{2} \in L(H, K V, \bar{V})$ then $H \cap K V \subset H_{2} \bar{V}$, indeed $H \cap K V \subset H_{2} \bar{V} \cap$ $K \bar{V}$ so that

$$
h \in H_{2} \bar{V} \cap K \bar{V}=\left(H_{2} \cap K \bar{V} \bar{V}^{-1}\right) \bar{V}
$$

hence

$$
h \in\left(U H_{1} \cap K \bar{V} \bar{V}^{-1}\right) \bar{V}=U\left(H_{1} \cap K \bar{V} \bar{V}^{-1}\right) \bar{V} .
$$

But this contradicts $U h \cap\left(H_{1} \cap K \bar{V} \bar{V}^{-1}\right) \bar{V}=\varnothing$, hence $R\left(H_{1}, K \bar{V} \bar{V}^{-1}, U\right) \subset$ $\mathscr{C} L(H, K V, \bar{V})$. Thus we have shown that $L(H, K V, \bar{V})$ is closed.

We next demonstrate that, under the further hypothesis that $V$ is symmetric, $(H, K V, \bar{V})$ is uniformly bounded, namely for $K^{\prime}$ compact and $U \in \mathscr{N}$ there exists a finite subset $\left\{H_{i}: i=1, \cdots, N\right\}$ of $L(H, K V, \bar{V})$ such that $\bigcup_{i=1}^{N} L\left(H_{i}, K^{\prime}, U\right) \supset L(H, K V, \bar{V})$.

It clearly suffices to assume that $K_{1} \supset K V \bar{V}^{-1}$ and $U$ is symmetric. Let $H \cap K V \bar{V}^{-1}=\left\{h_{1}, \cdots, h_{m}\right\}$. Each of the sets $h_{i} \bar{V}(i=1, \cdots, m)$ admits a finite covering of the form $\bigcup_{s=1}^{t_{i}} h_{i, s} U$ with $h_{i, s} \in h_{i} \bar{V}$ $\left(s=1, \cdots, t_{i} ; i=1, \cdots, m\right)$ and $K^{\prime} \backslash K V \bar{V}^{-1}$ admits a finite covering $\bigcup_{j=1}^{r} k_{j} U$ with $k_{j} \in K^{\prime} \backslash K V \bar{V}^{-1}(j=1, \cdots, r)$. We choose a finite set of elements $H^{\prime}$ as follows: $H^{\prime}$ is the union of non-empty, not necessarily proper, subsets of each of the sets $\left\{h_{i, s}: s=1, \cdots, t_{i}\right\}$ $(i=1, \cdots, m)$ together with a prossibly empty, not necessarily proper, subset of $\left\{k_{i}: i=1, \cdots, r\right\}$. We claim that these $\prod_{i=1}^{m}\left(2^{t_{i}}-1\right) \cdot 2^{r}$ sets satisfy the required condition. For the symmetry of $V$ ensures that each such set is in $L(H, K V, \bar{V})$; moreover if $H_{1} \in L(H, K V, \bar{V})$ then certainly there exists $H^{\prime}$ in our set for which $H_{1} \cap K^{\prime} \subset H^{\prime} U$. If we choose $H^{\prime}$ to be minimal in the sense that no proper subset of $H^{\prime}$ satisfies this condition we will ensure that $H^{\prime} \cap K^{\prime} \subset H_{1} U$ and hence $H_{1} \in L\left(H^{\prime}, K^{\prime}, U\right)$.

DeFinition 4. An equi-discrete subspace of $S(X)$ is one each member of which is a left $P$-packing for a fixed $P \in \mathscr{N}$. We denote this subspace by $S(X, P)$. 
Proposition 6. For each $P \in \mathscr{N}, S(X, P)$ is a closed subspace of $S(X)$.

Proof. Let $H \in \mathscr{C} S(X, P)$. Then there exists $x, y \in H, x \neq y$ such that $x P \cap y P \neq \varnothing$.

Choose $V_{1}=V_{1}^{-1} \in \mathscr{N}$ for which $x V_{1} \cap y V_{1}=\varnothing$ then $H^{\prime} \epsilon$ $L\left(H,\{x, y\}, V_{1}\right)$ implies that $\{x, y\} \subset H^{\prime} V_{1}$. Hence, if $x \in x^{\prime} V_{1}$ and $y \in y^{\prime} V_{1}$ for $x^{\prime}, y^{\prime} \in H^{\prime}$ then $x^{\prime} \neq y^{\prime}$.

Suppose that $z \in x P \cap y P$ then there exists a symmetric $W \in \mathscr{N}$ such that $W z \subset x P \cap y P$. Let $V_{2}=V_{2}^{-1} \in \mathscr{N}$ be such that $x V_{2} \subset W x$ and $V_{3}=V_{3}^{-1} \in \mathscr{N}$ satisfy y $V_{3} \subset W y$. With $V=V_{1} \cap V_{2} \cap V_{3}$ we have for $H^{\prime} \in L(H,\{x, y\}, V)$ that there exist distinct $x^{\prime}$ and $y^{\prime}$ in $H^{\prime}$ such that $x^{\prime} \in x V$ and $y^{\prime} \in y V$. Moreover since $x^{\prime} \in x V_{2}$ it follows that $x^{\prime}=w_{1} x$ for some $w_{1} \in W$. Then $x x^{\prime-1} z=w_{1}^{-1} z \in x P$ so $z \in x^{\prime} P$. Similarly $y^{\prime} \in y V_{3}$ implies that $y^{\prime} \in W y$ and we find that $z \in y^{\prime} P$. Thus $z \in x^{\prime} P \cap y^{\prime} P$ and we have shown that $L(H,\{x, y\}, V)$ is contained in $\mathscr{C} S(P, X)$ from which the proposition follows.

We conclude this paper with a proof of

THEOREM 3. If $X$ is $\sigma$-compact and satisfies the first axiom of countability then $S(P, X)$ is locally compact.

Proof. For any $H \in S(P, X)$ we set $L(P ; H, K, V)=L(H, K, V) \cap$ $S(P, X)$. We have that $L(P ; H, K V, \bar{V})$ is closed and for symmetric $V$ that $L(P ; H, K V, \bar{V})$ is uniformly bounded in $S(P, X)$ in that $L(H, K V, \bar{V})$ is uniformly bounded in $S(X)$. The theorem will follow upon showing that $S(P, X)$ is complete.

Let $\left(K_{i}\right)$ be a sequence of compact sets containing $e$ such that $K_{i+1} \supset K_{i}(i=1,2, \cdots)$ and $X=\bigcup_{i=1}^{\infty} K_{i}$. Let $\left(V_{j}\right)$ be a decreasing sequence of bounded, symmetric elements in $\mathscr{N}$ such that $\bigcap_{j=1}^{\infty}=$ $\{e\}$. If a sequence $\left(H_{n}\right)$ of elements in $\mathbf{S}(P, X)$ is Cauchy then for each $(i, j)$ there exists $n(i, j)$ such that, for all $n \geqq m \geqq n(i, j)$, $\left(H_{m}, H_{n}\right) \in p\left(K_{i}, V_{j}\right)$. It clearly suffices that this condition be met for all $(i, j)$ will $i=j$ and we shall write $n(i)$ for $n(i, i)$.

If $\left(H_{n}\right)=\{e\}$ for all $n>n_{0}$ then $H_{n}$ is certainly Cauchy and converges to $\{e\}$. Moreover if $\left(H_{n}\right)$ is Cauchy and $i$ is such that $H_{n(i)} \cap K_{i} V_{i}=\{e\}$ then $H_{m} \cap K_{i}=\{e\}$ for all $m \geqq n(i)$. If indeed $i$ is such that, for all $j \geqq i, H_{n(j)} \cap K_{j} V_{j}=\{e\}$ then again $\left(H_{n}\right)$ converges to $\{e\}$. It remains to consider $\left(H_{n}\right)$ for which this is not the case. Choose $i$ large enough that $V_{i} \subset P$. Then, for $n \geqq m \geqq n(i)$, $\left(H_{m}, H_{n}\right) \in p\left(K_{i}, V_{i}\right)$ and we may suppose $i$ large enough that $H_{m} \cap K_{i} \neq \varnothing$. If $h_{m} \in H_{m} \cap K_{i}$ then for each $n>m$ there exists 
$h_{n} \in H_{n}$ for which $h_{m} \in h_{n} V_{i}$. We claim that $h_{n}$ is uniquely determined for each $n>m$. For $h_{n}, h_{n}^{\prime} \in h_{m} V_{i}$ would imply that $h_{m} \in$ $h_{n} V_{i} \cap h_{n}^{\prime} V_{i}$ hence $h_{n} P \cap h_{n}^{\prime} P \neq \varnothing$ and therefore $h_{n}=h_{n}^{\prime}$. Thus for each $n>m$ there is a unique element in $H_{n} \cap K_{i} V_{i}$ which is contained in $h_{m} V_{i}$. We have, therefore, for each element $h_{m}$ in $H_{m} \cap K_{i}$ a sequence $\left\{h_{m, n}: n=1,2, \cdots\right\}$ which is moreover Cauchy in that for each $j>i$ there exists $n(j)$ such that, for $k>l>n(j), h_{m, k} h_{m, l}^{-1} \in$ $V_{j}$. Since $X$ is locally compact it is complete and therefore there exists a unique limit $\bar{h}_{m}$. Suppose that $\bar{h}_{m}$ and $\bar{h}_{m}^{\prime}$ are limits of two such sequences. We claim that $\bar{h}_{m} P \cap \bar{h}_{m}^{\prime} P=\varnothing$. Otherwise there would exist a symmetric neighborhood $V \in \mathscr{N}$ such that $V \bar{h}_{m}^{-1} \bar{h}_{m}^{\prime} V \subset$ $P P^{-1}$. If we take $s>i$ and large enough that $V_{s} \subset V$ then for $r>n(s)$ we would find $H_{r} \cap \bar{h}_{m} V_{s}=h_{r}$ and $H_{r} \cap \bar{h}_{m}^{\prime} V_{s}=h_{r}^{\prime}$ with $h_{r} \neq h_{r}^{\prime}$. Moreover $h_{r}^{-1} h_{r}^{\prime} \in P P^{-1}$ implying that $h_{r} P \cap h_{r}^{\prime} P \neq \varnothing$ contradicting $H_{r} \in S(P, X)$. It follows that the limit set $\bar{H}$ whose existence we have shown is an element of $S(P, X)$ and the proof is complete.

\section{REFERENCES}

1. C. Chabauty, Limites d' ensembles et geometrie des nombres, Bull. Soc. Math. France, 78 (1950), 143-151.

Received January 31, 1975 and in revised form September 17, 1975.

University of Pennsylvania 



\section{PACIFIC JOURNAL OF MATHEMATICS}

EDITORS

RICHARD ARENS (Managing Editor)

University of California

Los Angeles, California 90024

R. A. BEAUMONT

University of Washington

Seattle, Washington 98105
J. DugundJI

Department of Mathematics

University of Southern California

Los Angeles, California 90007

D. Gilbarg and J. Milgram

Stanford University

Stanford, California 94305

\section{ASSOCIATE EDITORS}

E. F. BECKENBACH

B. H. NeumanN

F. WOLF

K. YosHIDA

\section{SUPPORTING INSTITUTIONS}

UNIVERSITY OF BRITISH COLUMBIA

UNIVERSITY OF SOUTHERN CALIFORNIA

CALIFORNIA INSTITUTE OF TECHNOLOGY

UNIVERSITY OF CALIFORNIA

STANFORD UNIVERSITY

UNIVERSITY OF TOKYO

MONTANA STATE UNIVERSITY

UNIVERSITY OF UTAH

UNIVERSITY OF NEVADA

WASHINGTON STATE UNIVERSITY

NEW MEXICO STATE UNIVERSITY

UNIVERSITY OF WASHINGTON

OREGON STATE UNIVERSITY

UNIVERSITY OF OREGON

OSAKA UNIVERSITY

AMERICAN MATHEMATICAL SOCIETY
NAVAL WEAPONS CENTER

Printed in Japan by International Academic Printing Co., Ltd., Tokyo, Japan 


\section{Pacific Journal of Mathematics}

\section{Vol. 63, No. $1 \quad$ March, 1976}

Ralph Artino, Gevrey classes and hypoelliptic boundary value problems ....... 1

B. Aupetit, Caractérisation spectrale des algèbres de Banach commutatives .... 23

Leon Bernstein, Fundamental units and cycles in the period of real quadratic

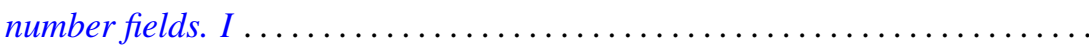

Leon Bernstein, Fundamental units and cycles in the period of real quadratic number fields. II.................................... 63

Robert F. Brown, Fixed points of automorphisms of compact Lie groups ........

Thomas Ashland Chapman, Concordances of noncompact Hilbert cube

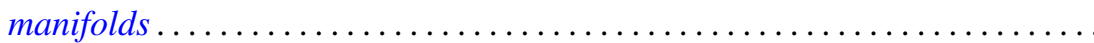

William C. Connett, V and Alan Schwartz, Weak type multipliers for Hankel

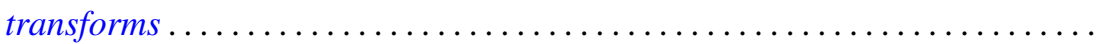

John Wayne Davenport, Multipliers on a Banach algebra with a bounded approximate identity .....................................

Gustave Adam Efroymson, Substitution in Nash functions ................ 137

John Sollion Hsia, Representations by spinor genera ..................

William George Kitto and Daniel Eliot Wulbert, Korovkin approximations in

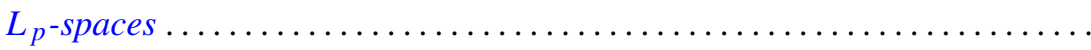

Eric P. Kronstadt, Interpolating sequences for functions satisfying a Lipschitz. condition ...........................................

Gary Douglas Jones and Samuel Murray Rankin, III, Oscillation properties of certain self-adjoint differential equations of the fourth order...

Takaŝi Kusano and Hiroshi Onose, Nonoscillation theorems for differential

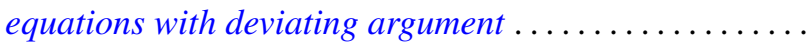

David C. Lantz, Preservation of local properties and chain conditions in commutative group rings. ...

Charles W. Neville, Banach spaces with a restricted Hahn-Banach extension property....

Norman Oler, Spaces of discrete subsets of a locally compact group ...

Robert Olin, Functional relationships between a subnormal operator and its minimal normal extension.

Thomas Thornton Read, Bounds and quantitative comparison theorems for nonoscillatory second order differential equations ...... .

Robert Horace Redfield, Archimedean and basic elements in completely distributive lattice-ordered groups...

Jeffery William Sanders, Weighted Sidon sets

Aaron R. Todd, Continuous linear images of pseudo-complete linear topological spaces.

J. Jerry Uhl, Jr., Norm attaining operators on $L^{1}[0,1]$ and the Radon-Nikodým property.

William Jennings Wickless, Abelian groups in which every endomorphism is a left multiplication. 